\title{
Accelerated phase contrast imaging using compressed sensing with complex difference sparsity
}

\author{
Yongjun Kwak ${ }^{1,2^{*}}$, Seunghoon Nam ${ }^{1,2}$, Kraig V Kissinger ${ }^{1}$, Beth Goddu', Lois A Goepfert', Warren J Manning ${ }^{1,3}$, \\ Vahid Tarokh², Reza Nezafat ${ }^{1}$
}

From 15th Annual SCMR Scientific Sessions

Orlando, FL, USA. 2-5 February 2012

\section{Background}

One of the major limitations of phase contrast imaging is its long scan time. Compressed sensing (CS) has been recently utilized to reduce the scan time in phase contrast MR [1-3]. In this study, we propose an accelerated phase contrast MR approach in which sparsity of the complex difference $(\mathrm{CD})$ image is used as the sparsifying transform to improve image reconstruction.

\section{Methods}

Figure 1 shows the proposed CS reconstruction with CD sparsity for phase contrast MR. The objective function $J$ $=\left\|F_{\Omega} m_{i}-y_{i}\right\|_{2}+\lambda\left\|\Psi m_{i}\right\|_{1}\left\|+\lambda{ }_{C D}\right\| m_{1}-m_{2} \|_{1}$, where $m_{i=1,2}$ are the two bipolar encoding acquisitions and $\left|m_{1}-m_{2}\right|$ is the corresponding $\mathrm{CD}$ image, is used in the reconstruction. The reconstruction performs iteratively between two bipolar images. Every iteration, the recon of $\mathrm{m}_{1}$ makes the intermediate image of $\mathrm{m}_{1}$ and pass it to the recon of $\mathrm{m}_{2}$, which uses it for calculating CD image and makes the intermediate image of $\mathrm{m}_{2}$ and pass it back to the recon of $m_{1}$. To evaluate the proposed method, phase contrast images were acquired using an axial slice of ascending aorta at the level of the bifurcation of the pulmonary artery. A retrospectively ECG-gated flowencoded 2D PC MRI pulse sequence was used with typical parameters of: $\mathrm{FOV}=320 \times 320 \mathrm{~mm}^{2}$, resolution $=$ $2.5 \mathrm{~ms} \times 2.5 \mathrm{~ms}$, slice thickness $=6 \mathrm{~mm}, \mathrm{TR} / \mathrm{TE}=15 /$ $6.5 \mathrm{~ms}$, flip angle $=30^{\circ}$, temporal resolution $=30 \mathrm{~ms}$, $\mathrm{VENC}=300 \mathrm{~cm} / \mathrm{s}$. The under-sampling was performed retrospectively from a fully sampled data using a Gaussian random under-sampling for rates of 3 and 5. In an

${ }^{1}$ Department of Medicine (Cardiovascular Division), Beth Israel Deaconess Medical Center, Boston, MA, USA

Full list of author information is available at the end of the article
IRB approved study, 14 healthy adult subjects (5 males, 17-70 years) were recruited. Reconstructed images were compared with fully-sampled acquisition and CS without CD sparsity (i.e. $\lambda_{C D}=0$ ).

\section{Results}

Figure 2a shows an example blood volume through the cardiac cycle for fully-sampled and under-sampled acquisition. Figure $2 b$ shows the linear regression relationship of blood volume between fully-sampled vs. under-sampled reconstruction for acceleration rate of 3 and 5. It shows that CS reconstruction with $C D$ gives the comparable volume measurements with the one with full-sampling. The corresponding Bland-Altman graphs are shown in Figure 2c and 2d, which shows excellent agreement between the two techniques.

\section{Conclusions}

In a retrospectively under-sampling study, accelerated CS with complex difference sparsity yields accurate flow measurement for acceleration rate of 3 and 5. Further study using a prospective accelerated acquisition in a larger patient cohort is needed to clinically evaluate this technique.

\section{Funding \\ NIH R01EB008743-01A2.}

\section{Author details}

'Department of Medicine (Cardiovascular Division), Beth Israel Deaconess Medical Center, Boston, MA, USA. ²Engineering Sciences, Harvard School of Engineering and Applied Sciences, Cambridge, MA, USA. ${ }^{3}$ Radiology, Beth Israel Deaconess Medical Center, Boston, MA, USA.

Published: 1 February 2012 


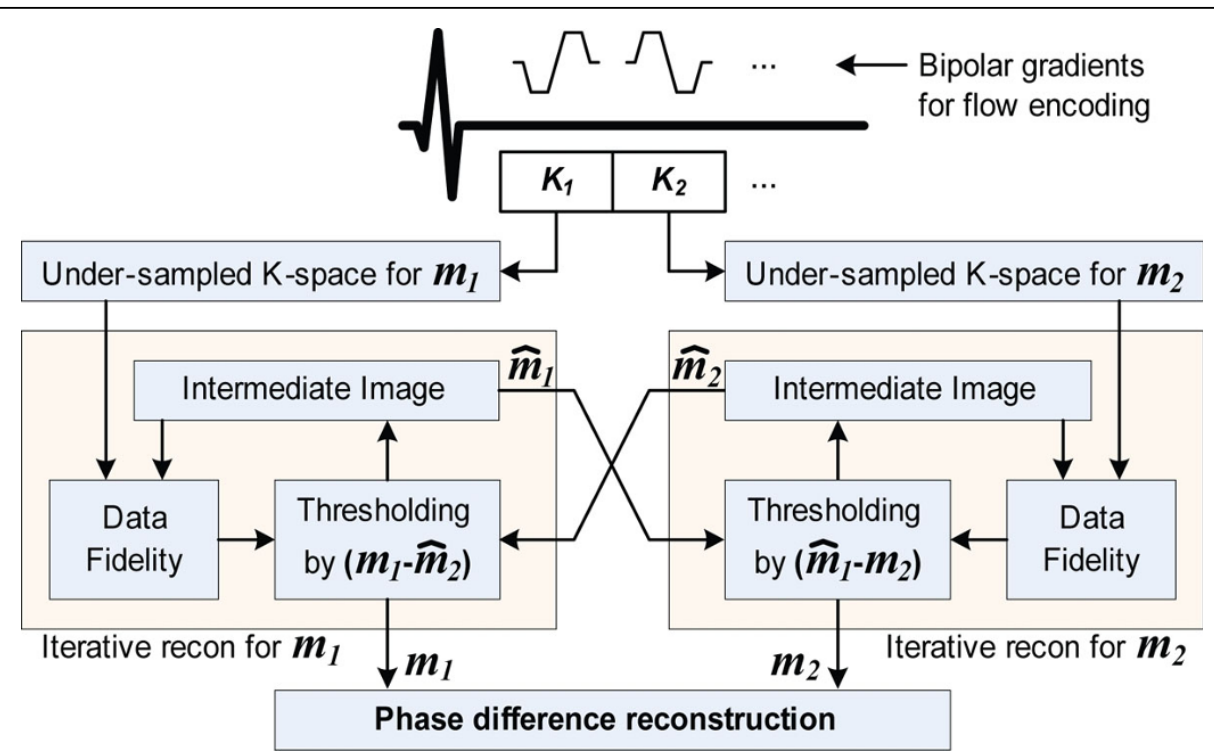

Figure 1 The proposed CS reconstruction algorithm for phase contrast MRI using complex-difference sparsity.
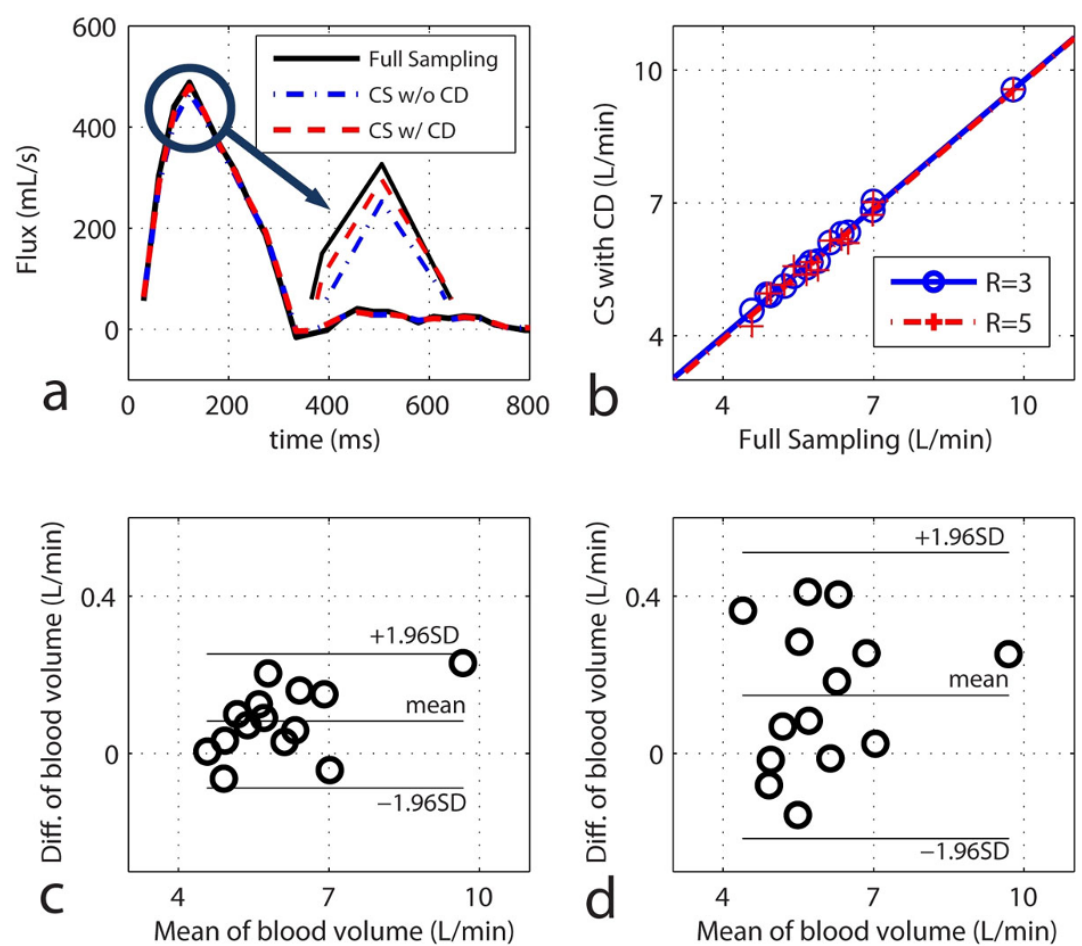

Figure 2 a) Example flow rate curves through ascending aorta for acceleration rate of 5, comparing the CS reconstruction method with and without CD sparsity. b) linear regression line between the CS reconstruction with CD sparsity and the reconstruction with full samples, $\mathbf{c}, \mathbf{d}$ ) Bland-Altman plots for rate 3 and 5 vs. fully sampled acquisition.

\section{References}

1. Kim : ISMRM. 2011

2. Hsiao : ISMRM. 2011

3. King : ISMRM. 2009.
doi:10.1186/1532-429X-14-S1-W24

Cite this article as: Kwak et al:: Accelerated phase contrast imaging

using compressed sensing with complex difference sparsity. Journal of Cardiovascular Magnetic Resonance 2012 14(Suppl 1):W24. 